\title{
Exact solutions for $(1+1)$-dimensional nonlinear dispersive modified Benjamin-Bona-Mahony equation and coupled Klein-Gordon equations
}

\author{
Kamruzzaman Khan ${ }^{1 *}$, M Ali Akbar² and S M Rayhanul Islam
}

\begin{abstract}
In this work, recently developed modified simple equation (MSE) method is applied to find exact traveling wave solutions of nonlinear evolution equations (NLEEs). To do so, we consider the (1 + 1)-dimensional nonlinear dispersive modified Benjamin-Bona-Mahony (DMBBM) equation and coupled Klein-Gordon (cKG) equations. Two classes of explicit exact solutions-hyperbolic and trigonometric solutions of the associated equations are characterized with some free parameters. Then these exact solutions correspond to solitary waves for particular values of the parameters.
\end{abstract}

Keywords: MSE method; NLEEs; DMBBM equation; CKG equation; Solitary wave; Exact solutions

PACS numbers: 02.30.Jr; 02.70.Wz; 05.45.Yv; 94.05.Fg

\section{Introduction}

The study of NLEEs, i.e., partial differential equations with time derivatives has a rich and long history, which has continued to attract attention in the last few decays. There are many examples throughout the world where NLEEs play an important role in controlling the natural systems. Because the majority of the phenomena in real world can be described by using NLEEs. NLEEs are frequently used to explain many problems of meteorology, population dynamics, fluid mechanics, plasma physics, optical fibers, biology, solid state physics, chemical kinematics, geochemistry, nanotechnology etc. By the aid of exact solutions, when they exist, the phenomena modeled by these NLEEs can be better understood. Therefore, the study of the traveling wave solutions for NLEEs plays an important role in the study of nonlinear physical phenomena.

In recent years, the exact solutions of NLEEs have been investigated by many authors who are interested in nonlinear physical phenomena. Many powerful methods have been presented by diverse group of mathematicians and physicists such as the Hirota's bilinear transformation method (Hirota 1973) (Hirota and Satsuma 1981),

\footnotetext{
* Correspondence: k.khanru@gmail.com

'Department of Mathematics, Pabna University of Science \& Technology,

Pabna 6600, Bangladesh

Full list of author information is available at the end of the article
}

the tanh-function method (Malfliet 1992; Nassar et al. 2011), the F-expansion method (Zhou et al. 2003), the $\left(G^{\prime} / G\right)$-expansion method (Wang et al. 2008; Zayed 2010; Zayed and Al-Joudi 2010, Zayed and Gepreel 2009; Akbar et al. 2012a, 2012b, 2012c, 2012d; Akbar and Ali 2011a; Shehata 2010; Zayed and Al-Joudi 2010; Naher et al. 2012a, 2013; Naher and Abdullah 2012, $2013)$, the enhanced $\left(G^{\prime} / G\right)$-expansion method (Khan et al. 2014, Khan and Akbar, 2014; Islam et al. 2014), the Exp-function method ( $\mathrm{He}$ and $\mathrm{Wu} 2006$; Bekir and Boz 2008; Akbar and Ali 2011b; Naher et al. 2011, 2012b; Yusufoglu 2008), the homogeneous balance method (Wang 1995; Zayed et al, 2004), the Adomian decomposition method (Adomian 1994), the homotopy perturbation method (Mohiud-Din 2007), the extended tanh-method (Abdou 2007; Fan 2000), the auxiliary equation method (Sirendaoreji 2004), the Jacobi elliptic function method (Ali 2011), Modified Exp-function method (He et al. 2012), the Modified simple equation method (Jawad et al. 2010; Zayed 2011; Zayed and Ibrahim 2012) and so on.

The purpose of this paper is to apply the MSE method to construct the exact solutions for nonlinear evolution equations in mathematical physics via the DMBBM equation and cKG equation. The DMBBM equation and cKG equation are NLEEs representing the balance of dispersion and weak nonlinearity in physical systems that generate solitary waves. 
The article is prepared as follows: The MSE method, Applications, Graphical representation of some obtained solutions, Comparisons, and conclusions.

\section{The MSE method}

Consider a general nonlinear evolution in the form

$$
\mathfrak{R}\left(u, u_{t}, u_{x}, u_{y}, u_{z}, u_{x x}, u_{t t}, u_{x z}, \ldots . .\right)=0
$$

where $\Re$ is a polynomial of $u(x, y, z, t)$ and its partial derivatives in which the highest order derivatives and nonlinear terms are involved. In the following, we give the main steps of this method (Jawad et al., 2010; Zayed, 2011, Zayed and Ibrahim, 2012):

Step 1. Using the traveling wave transformation

$$
u(x, y, z, t)=u(\xi), \quad \xi=x+y+z \pm \omega t,
$$

Eq. (2.1) transform to the following ODE:

$$
\wp\left(u, u^{\prime}, u^{\prime \prime}, \cdots\right)=0
$$

where $\wp$ is a polynomial in $u(\xi)$ and its derivatives, while $u^{\prime}(\xi)=\frac{d}{d \xi}, u^{\prime \prime}(\xi)=\frac{d^{2}}{d \xi^{2}}$, and so on.

Step 2. We suppose that Eq.(2.3) has the formal solution

$$
u(\xi)=\beta_{0}+\sum_{k=1}^{N} \beta_{k}\left[\frac{\Phi^{\prime}(\xi)}{\Phi(\xi)}\right]^{k}
$$

where $\beta_{k}$ are arbitrary constants to be determined, such that $\beta_{N} \neq 0$, and $\Phi(\xi)$ is an unknown function to be determined later.

Step 3. We determine the positive integer $N$ in Eq. (2.4) by considering the homogeneous balance between the highest order derivatives and the nonlinear terms in Eq. (2.3).

Step 4. We substitute (2.4) into (2.3), we calculate all the necessary derivatives $u^{\prime}, u^{\prime \prime}, \cdots$ and then we account the function $\Phi(\xi)$. As a result of this substitution, we get a polynomial of $\Phi^{\prime}(\xi) / \Phi(\xi)$ and its derivatives. In this polynomial, we equate with zero all the coefficients of $\Phi^{-i}(\xi)$, where $i=0,1,2, \cdots$. This operation yields a system of equations which can be solved to find $\beta_{k}$ and $\Phi(\xi)$. Consequently, we can get the exact solutions of Eq. (2.1).

\section{Applications}

The $(1+1)$-dimensional nonlinear dispersive modified Benjamin-Bona

Mahony equation: In this section, we will apply the modified simple equation method to find the exact solutions and then the solitary wave solutions of $(1+1)$ dimensional nonlinear DMBBM equation,

$$
u_{t}+u_{x}-\alpha u^{2} u_{x}+u_{x x x}=0
$$

where $\alpha$ is a nonzero constant. This equation was first derived to describe an approximation for surface long waves in nonlinear dispersive media. It can also characterize the hydro magnetic waves in cold plasma, acoustic waves in inharmonic crystals and acoustic gravity waves in compressible fluids (Yusufoglu 2008; Zayed and Al-Joudi 2010).

The traveling wave transformation is

$$
u=u(x, t), \xi=x-\omega t, u=u(\xi), u(x, t)=u(\xi)
$$

Using traveling wave Eq. (3.2), Eq. (3.1) transforms into the following ODE

$$
(1-\omega) u^{\prime}-\alpha u^{2} u^{\prime}+u^{\prime \prime \prime}=0
$$

Integrating with respect to $\xi$ choosing constant of integration as zero, we obtain the following ODE

$$
3(1-\omega) u-\alpha u^{3}+3 u^{\prime \prime}=0
$$

Now balancing the highest order derivative $u^{\prime \prime}$ and non-linear term $u^{3}$, we get

$3 N=N+2$, which gives $N=1$

Now for $N=1, u(\xi)=\beta_{0}+\sum_{k=1}^{N} \beta_{k}\left[\frac{\Phi^{\prime}(\xi)}{\Phi(\xi)}\right]^{k}$ becomes

$$
u(\xi)=\beta_{0}+\beta_{1}\left(\frac{\Phi^{\prime}}{\Phi}\right)
$$

where $\beta_{0}$ and $\beta_{1}$ are constants to be determined such that $\beta_{1} \neq 0$, while $\psi(\xi)$ is an unknown function to be determined. It is easy to see that

$$
\begin{aligned}
& u^{\prime \prime}=\beta_{1} \frac{\Phi^{\prime \prime \prime}}{\Phi}-3 \beta_{1} \frac{\Phi^{\prime \prime} \Phi^{\prime}}{\Phi^{2}}+2 \beta_{1}\left(\frac{\Phi^{\prime}}{\Phi}\right)^{3} \\
& u^{3}=\beta_{1}{ }^{3}\left(\frac{\Phi^{\prime}}{\Phi}\right)^{3}+3 \beta_{1}{ }^{2} \beta_{0}\left(\frac{\Phi^{\prime}}{\Phi}\right)^{2}+3 \beta_{1} \beta_{0}{ }^{2}\left(\frac{\Phi^{\prime}}{\Phi}\right)+\beta_{0}{ }^{3}
\end{aligned}
$$

Now substituting the values of $u^{\prime \prime}, u, u^{3}$ in equation (3.3) and then equating the coefficients of $\Phi^{0}, \Phi^{-1}, \Phi^{-2}, \Phi^{-3}$ to zero, we respectively obtain

$$
\begin{aligned}
& \alpha \beta_{0}{ }^{3}-3(1-\omega) \beta_{0}=0 \\
& \Phi^{\prime \prime \prime}-\left(\omega+\alpha \beta_{0}{ }^{2}-1\right) \Phi^{\prime}=0 \\
& 3 \Phi^{\prime \prime}+\alpha \beta_{0} \beta_{1} \Phi^{\prime}=0
\end{aligned}
$$

$$
\left(6 \beta_{1}-\alpha \beta_{1}^{3}\right) \Phi^{\prime 3}=0
$$


Solving Eq. (3.8), we get

$$
\beta_{0}=0, \pm \sqrt{\frac{3(1-\omega)}{\alpha}}
$$

Solving Eq. (3.11), we get

$\beta_{1}= \pm \sqrt{\frac{6}{\alpha}}$ and $\beta_{1} \neq 0$

Case I: when $\beta_{0}=0$ solving Eqs. (3.9), and (3.10) we get trivial solution. So this case is rejected.

Case II: when $\beta_{0}= \pm \sqrt{\frac{3(1-\omega)}{\alpha}}$, Eqs. (3.9) and (3.10) yields

$$
\Phi^{\prime \prime \prime}+l \Phi^{\prime \prime}=0
$$

where $l=\sqrt{(2(1-\omega))}$.

Integrating, Eq. (3.12) with respect to $\xi$, we obtain

$$
\Phi^{\prime \prime}=c_{1} \exp (-l \xi)
$$

From Eqs. (3.13) and (3.10), we obtain

$$
\Phi^{\prime}=\frac{-c_{1} \exp (-l \xi)}{\sqrt{(2(1-\omega))}}
$$

Therefore, upon integration, we obtain

$$
\Phi=c_{2}-\frac{c_{1} \exp (-l \xi)}{2(1-\omega)}
$$

where $c_{1}$ and $c_{2}$ are arbitrary constants.

Substituting the values of $\Phi$ and $\Phi^{\prime}$ into Eq. (3.5), we obtain the following exact solution,

$$
u(\xi)=\beta_{0}+\beta_{1} \frac{-2(1-\omega) c_{1} \exp (-l \xi)}{\sqrt{2(1-\omega)}\left(2(1-\omega) c_{2}+c_{1} \exp (-l \xi)\right)}
$$

Putting the values of $\beta_{0}, \beta_{1}, l$ and simplifying, we obtain

$$
\begin{aligned}
u(x, t)= & \pm \sqrt{\frac{3(1-\omega)}{\alpha}} \\
& \left(\begin{array}{c}
2 c_{1}\left(\begin{array}{c}
\cosh \left(\sqrt{\left(\frac{(1-\omega)}{2}\right)}(x-\omega t)\right) \\
-\sinh \left(\sqrt{\left(\frac{(1-\omega)}{2}\right)}(x-\omega t)\right)
\end{array}\right) \\
\left.1-\frac{\left(2(1-\omega) c_{2}-c_{1}\right) \cosh \left(\sqrt{\left(\frac{(1-\omega)}{2}\right)}(x-\omega t)\right)}{+\left(2(1-\omega) c_{2}+c_{1}\right) \sinh \left(\sqrt{\left(\frac{(1-\omega)}{2}\right)}(x-\omega t)\right)}\right)
\end{array}\right)
\end{aligned}
$$

Since $c_{1}$ and $c_{2}$ are arbitrarily constants, consequently, if we set $c_{1}=-2 c_{2}(1-\omega)$ and $\frac{(1-\omega)}{2}>0$, Eq. (3.17) reduces to the following traveling wave solution:

$$
u_{1,2}(x, t)= \pm \sqrt{\left(\frac{3(1-\omega)}{\alpha}\right)} \tanh \left(\sqrt{\left(\frac{(1-\omega)}{2}\right)}(x-\omega t)\right)
$$

Again setting $c_{1}=2 c_{2}(1-\omega)$ and if $\frac{(1-\omega)}{2}>0$, Eq. (3.17) reduces to the following singular traveling wave solutions:

$$
u_{3,4}(x, t)= \pm \sqrt{\left(\frac{3(1-\omega)}{\alpha}\right)} \operatorname{coth}\left(\sqrt{\left(\frac{(1-\omega)}{2}\right)}(x-\omega t)\right)
$$

If $\frac{(1-\omega)}{2}<0$, Eqs. (3.18) and (3.19) yields the following periodic solutions:

$$
u_{5,6}(x, t)= \pm \sqrt{\left(\frac{3(\omega-1)}{\alpha}\right)} \tan \left(\sqrt{\left(\frac{(\omega-1)}{2}\right)}(x-\omega t)\right)
$$

and

$$
u_{7,8}(x, t)= \pm \sqrt{\left(\frac{3(\omega-1)}{\alpha}\right)} \cot \left(\sqrt{\left(\frac{(\omega-1)}{2}\right)}(x-\omega t)\right) .
$$

Remark 1: From solutions (3.18)-(3.21) we conclude that $\omega \neq 1$.

\section{The coupled Klein-Gordon equation}

Now we will bring to bear the MSE method to find exact solutions and then the solitary wave solutions to the cKG Equation in the form,

$$
\begin{aligned}
& u_{x x}-u_{t t}-u+2 u^{3}+2 u v=0 \\
& v_{x}-v_{t}-4 u u_{t}=0
\end{aligned}
$$

where

$$
u(\xi)=u(x, t), v(\xi)=v(x, t), \xi=x-\omega t .
$$

The traveling wave Eq. (3.23) reduces Eqs. (3.22) into the following ODEs

$$
\begin{aligned}
& \left(1-\omega^{2}\right) u^{\prime \prime}-u+2 u^{3}+2 u v=0 \\
& (1+\omega) v^{\prime}+4 \omega u u^{\prime}=0
\end{aligned}
$$

By integrating Eq. (3.25) with respect to $\mathcal{\xi}$, and neglecting the constant of integration we obtain

$$
v=-\frac{2 \omega}{1+\omega} u^{2}
$$


Substituting Eq. (3.26) into Eq. (3.24) we get,

$$
\left(1-\omega^{2}\right) u^{\prime \prime}-u+\frac{2(1-\omega)}{1+\omega} u^{3}=0 .
$$

Balancing the highest order derivative $u^{\prime \prime}$ and nonlinear term $u^{3}$ from Eq. (3.27), we obtain $N=1$

Now for $N=1$, Eq. (2.4) becomes

$$
u(\xi)=\beta_{0}+\beta_{1}\left(\frac{\Phi^{\prime}}{\Phi}\right)
$$

where $\beta_{0}$ and $\beta_{1}$ are constants to be determined such that $\beta_{1} \neq 0$, while $\Phi(\xi)$ is an unknown function to be determined. It is easy to see that

$$
\begin{aligned}
& u^{\prime \prime}=\beta_{1} \frac{\Phi^{\prime \prime \prime}}{\Phi}-3 \beta_{1} \frac{\Phi^{\prime \prime} \Phi^{\prime}}{\Phi^{2}}+2 \beta_{1}\left(\frac{\Phi^{\prime}}{\Phi}\right)^{3} \\
& u^{3}=\beta_{1}{ }^{3}\left(\frac{\Phi^{\prime}}{\Phi}\right)^{3}+3 \beta_{1}{ }^{2} \beta_{0}\left(\frac{\Phi^{\prime}}{\Phi}\right)^{2}+3 \beta_{1} \beta_{0}{ }^{2}\left(\frac{\Phi^{\prime}}{\Phi}\right)+\beta_{0}{ }^{3}
\end{aligned}
$$

Now substituting the values of $u^{\prime \prime}, u, u^{3}$ in Eq. (3.27) and then equating the coefficients of $\Phi^{0}, \Phi^{-1}, \Phi^{-2}, \Phi^{-3}$ to zero, we respectively obtain

$$
\begin{aligned}
& \beta_{0}^{3}\left(\frac{2}{1+\omega}-\frac{2 \omega}{1+\omega}\right)-\beta_{0}=0 \\
& \left(1-\omega^{2}\right) \Phi^{\prime \prime}-\left(\frac{2 \beta_{0} \beta_{1}(1-\omega)}{1+\omega}\right) \Phi^{\prime}=0 \\
& \left(1-\omega^{2}\right) \Phi^{\prime \prime \prime}+\left(\frac{6 \beta_{0}^{2}(1-\omega)}{1+\omega}-1\right) \Phi^{\prime}=0 \\
& -2 \omega^{2} \beta_{1}+2 \beta_{1}+\frac{2 \beta_{1}^{3}}{1+\omega}-\frac{2 \omega \beta_{1}^{3}}{1+\omega}=0 .
\end{aligned}
$$

Solving Eq. (3.31), we get

$$
\beta_{0}=0, \pm \sqrt{\left(\frac{1+\omega}{2(1-\omega)}\right)}
$$

Solving Eq. (3.34), we get

$$
\beta_{1}= \pm I(1+\omega) \text { and } \beta_{1} \neq 0, I=\sqrt{-1}
$$

Case-I: When $\beta_{0}=0$, Eq. (3.32) and (3.33) yields a trivial solution. So this case is rejected.

Case-II: When $\beta_{0}= \pm \sqrt{\frac{(1+\omega)}{2(1-\omega)}}$, Eqs. (3.32) and (3.33) yields,

$$
\Phi^{\prime \prime \prime}-I \sqrt{\left(\frac{1-\omega^{2}}{2}\right)} \Phi^{\prime \prime}=0
$$

Integrating, Eq. (3.35) with respect to $\xi$, we obtain

$$
\Phi^{\prime \prime}=c_{1} \exp \left(I \sqrt{\left(\frac{2}{1-\omega^{2}}\right)} \xi\right)
$$

From Eqs. (3.36) and (3.32), we obtain

$$
\Phi^{\prime}=-I \sqrt{\left(\frac{1-\omega^{2}}{2}\right)} c_{1} \exp \left(I \sqrt{\left(\frac{2}{1-\omega^{2}}\right)} \xi\right) .
$$

Therefore, integration Eq. (3.37), we obtain

$$
\Phi=\frac{1}{2}\left(2 c_{2}-\left(1-\omega^{2}\right) c_{1} \exp \left(I \sqrt{\left(\frac{2}{1-\omega^{2}}\right)} \xi\right)\right)
$$

where $c_{1}$ and $c_{2}$ are constants of integration.

Substituting the values of $\Phi$ and $\Phi^{\prime}$ into Eq. (3.28), we obtain the following exact solution,

$$
u(\xi)=\beta_{0}-\beta_{1} \frac{I \sqrt{2\left(1-\omega^{2}\right)} c_{1} \exp \left(I \sqrt{\left(\frac{2}{1-\omega^{2}}\right)} \xi\right)}{2 c_{2}-\left(1-\omega^{2}\right) c_{1} \exp \left(I \sqrt{\left(\frac{2}{1-\omega^{2}}\right)} \xi\right)} .
$$

Putting the values of $\beta_{0}, \beta_{1}$ into Eq. (3.39) and then simplifying, we obtain

$$
\begin{aligned}
u(x, t)= & \sqrt{\left(\frac{1+\omega}{2(1-\omega)}\right)} \\
& \left(\begin{array}{c}
2\left(1-\omega^{2}\right) c_{1}\left(\begin{array}{c}
\cosh \left(\frac{I}{\sqrt{2\left(1-\omega^{2}\right)}}(x-\omega t)\right) \\
+\sinh \left(\frac{I}{\sqrt{2\left(1-\omega^{2}\right)}}(x-\omega t)\right)
\end{array}\right) \\
\left.1+\frac{\left(2 c_{2}-\left(1-\omega^{2}\right) c_{1}\right) \cosh \left(\frac{I}{\sqrt{2\left(1-\omega^{2}\right)}}(x-\omega t)\right)}{-\left(2 c_{2}-\left(1-\omega^{2}\right) c_{1}\right) \sinh \left(\frac{I}{\sqrt{2\left(1-\omega^{2}\right)}}(x-\omega t)\right)}\right)
\end{array}\right) .
\end{aligned}
$$

We can freely choose the constants $c_{1}$ and $c_{2}$. Therefore, if we set $c_{2}=\frac{\left(1-\omega^{2}\right) c_{1}}{2}$, Eq. (3.40) reduces to:

$$
u_{1,2}(x, t)= \pm \sqrt{\left(\frac{1+\omega}{2(1-\omega)}\right)} \operatorname{coth}\left(\frac{I(x-\omega t)}{\sqrt{2\left(1-\omega^{2}\right)}}\right)
$$




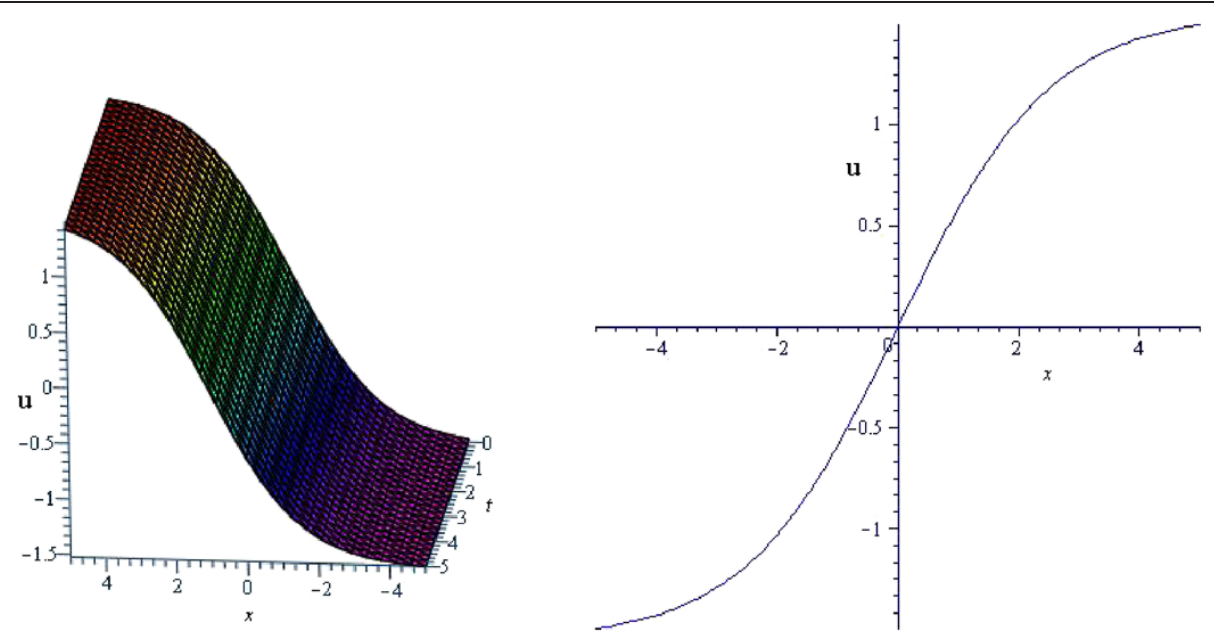

Figure 1 Kink (topological soliton) profile of DMBBM equation for $\boldsymbol{\omega}=\mathbf{0 . 2 0 ,} \boldsymbol{a}=\mathbf{1}$. (Only shows the shape of (3.18), The left figure shows the 3-D plot and the right figure shows the 2-D plot for $t=0$.

Again, if we set $c_{2}=-\frac{\left(1-\omega^{2}\right) c_{1}}{2}$, Eq. (3.40) reduces to:

$$
u_{3,4}(x, t)= \pm \sqrt{\left(\frac{1+\omega}{2(1-\omega)}\right)} \tanh \left(\frac{I(x-\omega t)}{\sqrt{2\left(1-\omega^{2}\right)}}\right) .
$$

Using hyperbolic identities, in trigonometric form Eqs. (3.41) and (3.42) can be written as follows:

$$
\begin{aligned}
& u_{5,6}(x, t)=\mp I \sqrt{\left(\frac{1+\omega}{2(1-\omega)}\right)} \cot \left(\frac{(x-\omega t)}{\sqrt{2\left(1-\omega^{2}\right)}}\right), \\
& u_{7,8}(x, t)= \pm I \sqrt{\left(\frac{1+\omega}{2(1-\omega)}\right)} \tan \left(\frac{(x-\omega t)}{\sqrt{2\left(1-\omega^{2}\right)}}\right) .
\end{aligned}
$$

Now applying Eqs. (3.41)-(3.44) into Eq. (3.26), we get

$$
\begin{aligned}
& v_{1}(x, t)=\left(\frac{\omega}{1-\omega}\right) \operatorname{coth}^{2}\left(\frac{I(x-\omega t)}{\sqrt{2\left(1-\omega^{2}\right)}}\right) \\
& v_{2}(x, t)=\left(\frac{\omega}{1-\omega}\right) \tanh ^{2}\left(\frac{I(x-\omega t)}{\sqrt{2\left(1-\omega^{2}\right)}}\right) \\
& v_{3}(x, t)=\left(\frac{\omega}{1-\omega}\right) \cot ^{2}\left(\frac{(x-\omega t)}{\sqrt{2\left(1-\omega^{2}\right)}}\right) \\
& v_{4}(x, t)=\left(\frac{\omega}{1-\omega}\right) \tan ^{2}\left(\frac{(x-\omega t)}{\sqrt{2\left(1-\omega^{2}\right)}}\right)
\end{aligned}
$$

Remark 2: From solutions (3.41)-(3.48) we conclude that $\omega \neq \pm 1$.
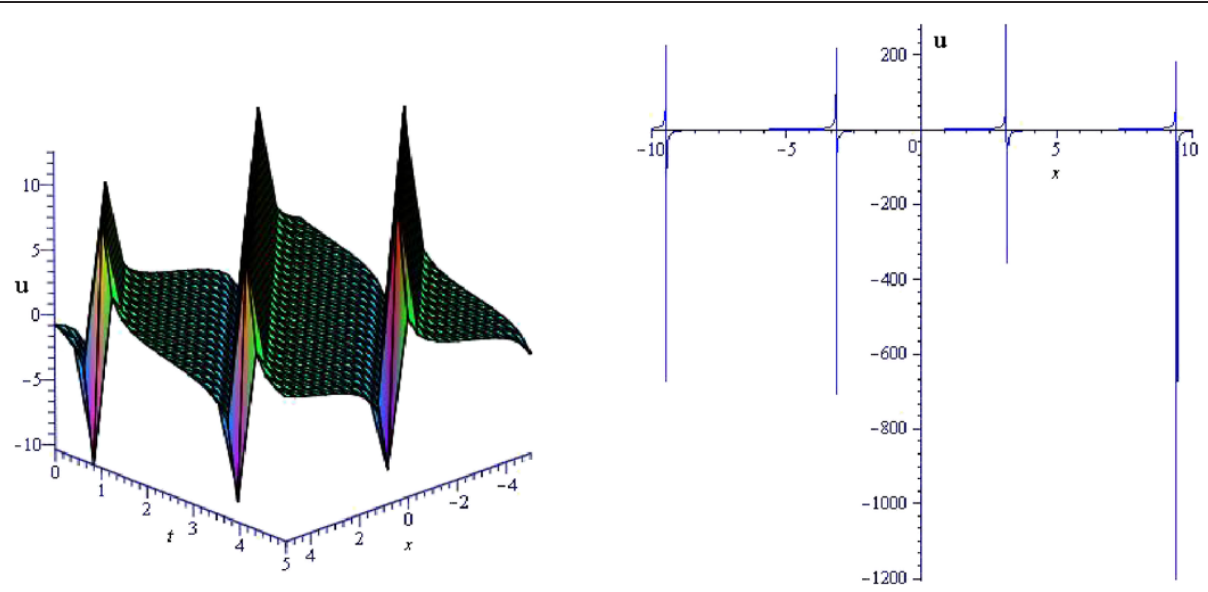

Figure 2 Periodic graph of DMBBM equation for $\boldsymbol{\omega}=\mathbf{2}, \boldsymbol{a}=\mathbf{3}$. (Only shows the shape of (3.20)), The left figure shows the 3-D plot and the right figure shows the $2-D$ plot for $t=0$. 

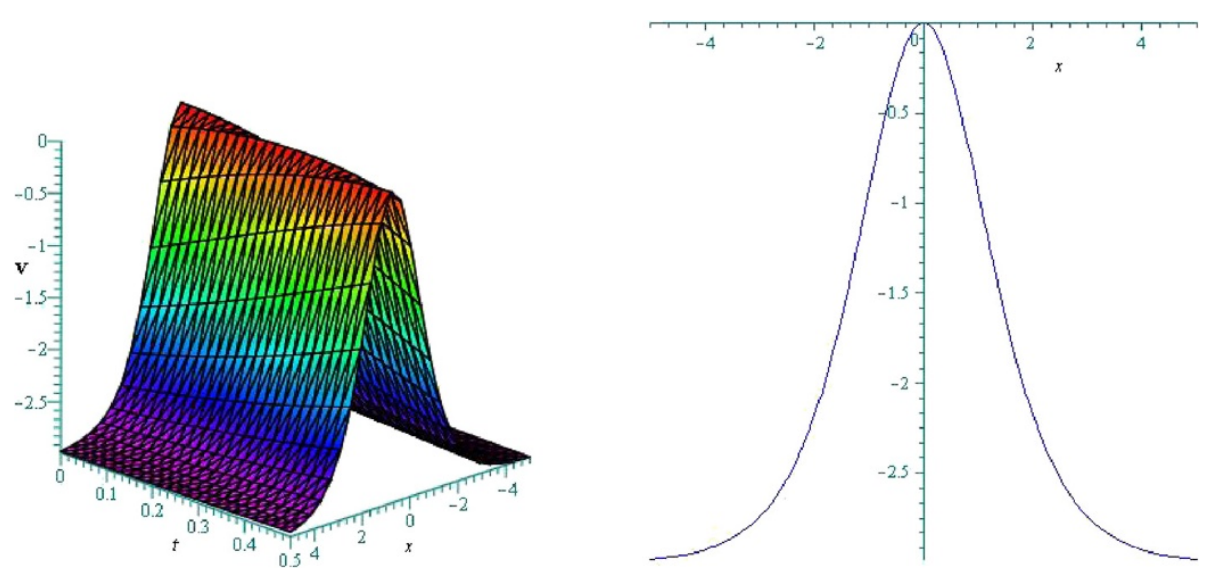

Figure 3 Bell (non- topological soliton) profile of cKG equation for $\boldsymbol{\omega}=\mathbf{1 . 5 0}$. (Only shows the shape of solution (3.46)), The left figure shows the 3-D plot and the right figure shows the 2-D plot for $t=0$.

\section{Graphical representation of some obtained solutions}

In this section, we put forth to illustrate the threedimensional and two-dimensional structure of the determined solutions of the studied NLEEs, to visualize the inner mechanism of them.

Figure 1 and Figure 2 represent the shape of solutions (3.18) and (3.20) of DMBBM equation. On the other hand, Figure 3 and Figure 4 show the profile of solutions (3.46) and (3.48) of cKG equation.

\section{Comparisons}

\section{With extended $\left(G^{\prime} / G\right)$-expansion method:}

Zayed and Al-Joudi (2010) investigated exact solutions of the traveling wave solutions of the DMBBM equation by using the extended $\left(G^{\prime} / G\right)$-expansion method and obtained six solutions. On the contrary by using the MSE method in this article we obtained eight solutions.
However, Some of the solutions obtained by Zayed and Al-Joudi (2010) coincide with our solutions. If we set $\omega=$ $1+2 \mu$ in our solutions (3.18) and (3.19), we conclude that our results coincide to the solution (3.9) obtained by Zayed and Al-Joudi (2010) for $A \neq 0, B=0, \mu<0, \sigma= \pm 1$ and $A=0, B \neq 0, \mu<0, \sigma= \pm 1$ respectively. Similarly, solutions (3.20) and (3.21) obtained in this article correspond to the solution (3.12) obtained by Zayed and Al-Joudi (2010) for $A \neq 0, B=0, \mu>0, \sigma= \pm 1$ and $A=0, B \neq 0, \mu>0$, $\sigma= \pm 1$ respectively.

Moreover, Zayed and Al-Joudi (2010) used the symbolic computation software such as Maple or Mathematica to facilitate the calculation of the algebraic equations occurred in the solution procedure. Without symbolic computation software even it is impossible to get the solutions of the complicated algebraic equations. In addition, Zayed and Al-Joudi (2010) used the solutions of an auxiliary equation $G^{\prime \prime}(\xi)+\mu G(\xi)=0$ to find exact traveling wave
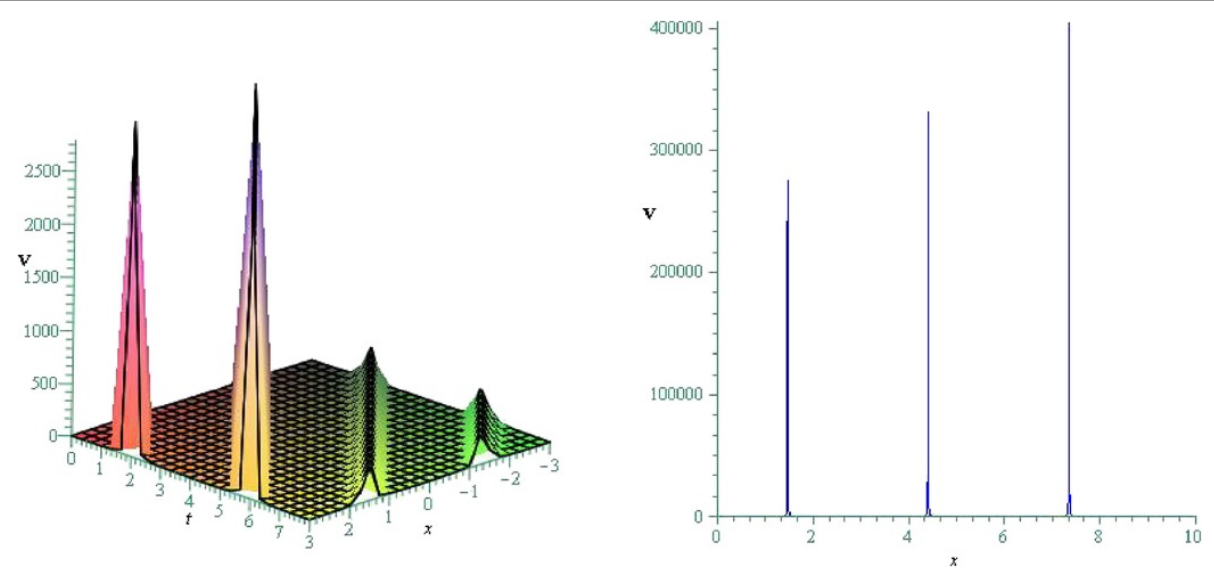

Figure 4 Periodic profile of $\mathbf{c K G}$ equation for $\boldsymbol{\omega}=\mathbf{0 . 7 5}$. (Only shows the shape of (3.48)), The left figure shows the 3-D plot and the right figure shows the 2-D plot for $t=0$. 
solutions of NLEEs. On the other hand it is worth mentioning that the exact solutions of the studied NLEEs have been achieved in this article without using any symbolic computations software, since the computations are very simple and easy. Similarly for any nonlinear evolution equation it can be shown that the MSE method is much easier than other methods. Furthermore, auxiliary equations are unnecessary to solve NLEEs by means of MSE methods, i.e., there exists no predefined functions or equations in MSE method.

\section{Conclusions}

This study shows that the MSE method is quite efficient and practically well suited for use to find exact traveling wave solutions of the DMBBM equation and CKG equation. We have obtained exact solutions of these equations in terms of the hyperbolic and trigonometric functions. This study also shows that the procedure is simple, direct and constructive. The reliability of the method and the reduction in the size of computational domain give this method a wider applicability. We conclude that the studied method can be used for many other NLEEs in mathematical physics and engineering fields.

\section{Competing interests}

The authors declare that they have no competing interests.

\section{Authors' contributions}

This work was carried out in collaboration among the authors. All authors have a good contribution to design the study, and to perform the analysis of this research work. All authors read and approved the final manuscript.

\section{Author details}

'Department of Mathematics, Pabna University of Science \& Technology, Pabna 6600, Bangladesh. ${ }^{2}$ Department of Applied Mathematics, University of Rajshahi, Rajshahi 6205, Bangladesh.

Received: 27 August 2014 Accepted: 20 November 2014 Published: 10 December 2014

\section{References}

Abdou MA (2007) The extended tanh-method and its applications for solving nonlinear physical models. App Math Comput 190:988-996

Adomian G (1994) Solving frontier problems of physics: The decomposition method. Kluwer Academic, Boston, M A

Akbar MA, Ali NHM, Zayed EME (2012a) Abundant exact traveling wave solutions of the generalized Bretherton equation via $\left(\mathrm{G}^{\prime} / \mathrm{G}\right)$-expansion method. Commun Theor Phys 57:173-178

Akbar MA, Ali NHM, Zayed EME (2012b) A generalized and improved (G'/G) expansion method for nonlinear evolution equations. Math Prob Engr 2012:22, doi:10.1155/2012/459879

Akbar MA, Ali NHM, Mohyud-Din ST (2012C) The alternative ( $\left.G^{\prime} / G\right)$ - expansion method with generalized Riccati equation: Application to fifth order $(1+1)$ dimensional Caudrey-Dodd-Gibbon equation. Int J Phys Sci 7(5):743-752

Akbar MA, Ali NHM, Mohyud-Din ST (2012d) Some new exact traveling wave solutions to the $(3+1)$-dimensional Kadomtsev-Petviashvili equation. World Appl Sci J 16(11):1551-1558

Akbar MA, Ali NHM (2011a) The alternative $\left(\mathrm{G}^{\prime} / \mathrm{G}\right)$-expansion method and its applications to nonlinear partial differential equations. Int J Phys Sci 6(35):7910-7920

Akbar MA, Ali NHM (2011b) Exp-function method for Duffing Equation and new solutions of $(2+1)$ dimensional dispersive long wave equations. Prog Appl Math 1(2):30-42
Ali AT (2011) New generalized Jacobi elliptic function rational expansion method. J Comput Appl Math 235:4117-4127

Bekir A, Boz A (2008) Exact solutions for nonlinear evolution equations using Exp- function method. Phys Lett 372:1619-1625

Fan EG (2000) Extended tanh-method and its applications to nonlinear equations. Phys Lett 277:212-218

He Y, Li S, Long Y (2012) Exact solutions of the Klein-Gordon equation by modified Exp-function method. Int Math Forum 7(4):175-182

He JH, Wu XH (2006) Exp-function method for nonlinear wave equations. Chaos, Solitons and Fract 30:700-708

Hirota R (1973) Exact envelope soliton solutions of a nonlinear wave equation. J Math Phy 14:805-810

Hirota R, Satsuma J (1981) Soliton solutions of a coupled KDV equation. Phy Lett A 85:404-408

Islam MH, Khan K, Akbar MA, Salam MA (2014) Exact Traveling Wave Solutions of Modified KdV-Zakharov-Kuznetsov Equation and Viscous Burgers Equation. SpringerPlus 3:105, doi:10.1186/2193-1801-3-105

Jawad AJM, Petkovic MD, Biswas A (2010) Modified simple equation method for nonlinear evolution equations. Appl Math Comput 217:869-877

Khan K, Akbar MA, Salam MA, Islam MH (2014) A note on enhanced ( $\left.G^{\prime} / G\right)$ expansion method in nonlinear physics. Ain Shams Engineering Journal 5:877-884, http://dx.doi.org/10.1016/j.asej.2013.12.013

Khan K, Akbar MA (2014) Study of analytical method to seek for exact solutions of variant Boussinesq equations. SpringerPlus 3:324, doi:10.1186/21931801-3-324

Malfliet M (1992) Solitary wave solutions of nonlinear wave equations. Am J Phys 60:650-654

Mohiud-Din ST (2007) Homotopy perturbation method for solving fourth-order boundary value problems. Math Prob Engr 2007:1-15, Article ID 98602, doi:10.1155/2007/98602

Naher H, Abdullah AF, Akbar MA (2012a) New traveling wave solutions of the higher dimensional nonlinear partial differential equation by the Exp-function method. J Appl Math, Article ID 575387, 14 pages. doi:10.1155/ 2012/575387

Naher H, Abdullah AF, Akbar MA (2011) The Exp-function method for new exact solutions of the nonlinear partial differential equations. Int J Phys Sci 6(29):6706-6716

Naher $\mathrm{H}$, Abdullah FA, Bekir A (2012b) Abundant traveling wave solutions of the compound KdV-Burgers equation via the improved $\left(\mathrm{G}^{\prime} / \mathrm{G}\right)$-expansion method. AIP Adv, 2, 042163. http://dx.doi.org/10.1063/1.4769751

Naher H, Abdullah FA, Akbar MA (2013) Generalized and improved (G'/G)expansion method for $(3+1)$-dimensional modified KdV-ZakharovKuznetsev equation. PLoS One 8(5):e64618

Naher $\mathrm{H}$, Abdullah FA (2012) Some new traveling wave solutions of the nonlinear reaction diffusion equation by using the improved $\left(\mathrm{G}^{\prime} / \mathrm{G}\right)$-expansion method. Math Prob Engr 2012:17, http://dx.doi.org/10.1155/2012/871724

Naher $H_{1}$ Abdullah FA (2013) New approach of ( $\left.G^{\prime} / G\right)$-expansion method and new approach of generalized $\left(G^{\prime} / G\right)$-expansion method for nonlinear evolution equation. AIP Adv 3(3):032116

Nassar HA, Abdel-Razek MA, Seddeek AK (2011) Expanding the tanh-function method for solving nonlinear equations. Appl Math 2:1096-1104

Shehata AR (2010) The traveling wave solutions of the perturbed nonlinear Schrodinger equation and the cubic-quintic Ginzburg Landau equation using the modified ( $\mathrm{G}^{\prime} / \mathrm{G}$ )-expansion method. Appl Math Comput 217:1-10

Sirendaoreji (2004) New exact travelling wave solutions for the Kawahara and modified Kawahara equations. Chaos Solitons Fract 19:147-150

Wang M (1995) Solitary wave solutions for variant Boussinesq equations. Phys Lett 199:169-172

Wang M, Li X, Zhang J (2008) The (G'/G)-expansion method and travelling wave solutions of nonlinear evolution equations in mathematical physics. Phys Lett 372:417-423

Yusufoglu $E$ (2008) New solitary solutions for the MBBM equations using Exp-function method. Phys Lett 372:442-446

Zayed EME (2011) A note on the modified simple equation method applied to Sharma- Tasso-Olver equation. Appl Math Comput 218:3962-3964

Zayed EME, Ibrahim SAH (2012) Exact solutions of nonlinear evolution equations in Mathematical physics using the modified simple equation method. Chinese Phys Lett 29(6):060201

Zayed EME, Zedan HA, Gepreel KA (2004) On the solitary wave solutions for nonlinear Hirota-Sasuma coupled KDV equations. Chaos, Solitons and Fractals 22:285-303 
Zayed EME, Al-Joudi S (2010) Applications of an extended (G'/G)-expansion method to find exact solutions of nonlinear PDEs in mathematical physics. Math Prob Engr, 19 pages, doi:10.1155/2010/768573

Zayed EME (2010) Traveling wave solutions for higher dimensional nonlinear evolution equations using the (G'/G)-expansion method. J Appl Math Informatics 28:383-395

Zayed EME, Gepreel KA (2009) The (G'/G)-expansion method for finding the traveling wave solutions of nonlinear partial differential equations in mathematical physics. J Math Phys 50:013502-013514

Zhou YB, Wang ML, Wang YM (2003) Periodic wave solutions to coupled KdV equations with variable coefficients. Phys Lett 308:31-36

doi:10.1186/2193-1801-3-724

Cite this article as: Khan et al:: Exact solutions for $(1+1)$-dimensional nonlinear dispersive modified Benjamin-Bona-Mahony equation and coupled Klein-Gordon equations. SpringerPlus 2014 3:724.

\section{Submit your manuscript to a SpringerOpen ${ }^{\circ}$ journal and benefit from:}

- Convenient online submission

- Rigorous peer review

- Immediate publication on acceptance

- Open access: articles freely available online

- High visibility within the field

- Retaining the copyright to your article

Submit your next manuscript at $>$ springeropen.com 